
\title{
25 Research Soure \\ Mast Cells and COVID-19: a case report implicating a role of mast cell activation in the prevention and treatment of Covid-19
}

Isabelle Brock ( $\square$ research@connective-tissue-issues.com )

Indiana University https://orcid.org/0000-0003-4144-9763

Anne Maitland

Icahn School of Medicine

\section{Case Report}

Keywords: coronavirus, SARS CoV2, COVID-19, mast cell, mast cell activation syndrome, tryptase, case report

Posted Date: March 15th, 2021

DOl: https://doi.org/10.21203/rs.3.rs-330667/v1

License: (c) (i) This work is licensed under a Creative Commons Attribution 4.0 International License.

Read Full License 


\section{Abstract}

Coronavirus disease (COVID-19) is a heterogeneous syndrome following severe acute respiratory syndrome coronavirus 2 (SARS-CoV-2) infection of the upper respiratory tract. In adults, the clinical condition can range from asymptomatic cases to severe acute respiratory syndrome and multi-organ dysfunction. Those at risk of developing COVID-19 related hyperinflammatory syndrome likely had an ineffective, innate immune response to this novel pathogen. Mast cells are associated with the epithelium, contributing to tissue homeostasis and epithelial barrier defense. Equipped with an array of pathogen receptors, mast cells exhibit distinct cytokine profiles, dependent on the tissue and the triggered pathogen receptors. Following viral infections, mast cells produce pro-inflammatory chemical mediators, such as interleukin-1 (IL-1) and IL-6, and these cytokines has been shown to be elevated in severe COVID19 cases. Here, we present a case of a patient with a longstanding history of signs and symptoms, worrisome for a mast cell activation syndrome (MCAS), but never had laboratory confirmation of this non-clonal mast cell activation disorder, until she contracted COVID-19. This case illustrates the need to recognize the rate of mast cell activation in SARS-CoV-2 infection, not only to optimize anti-SARS-CoV-2 therapy, including the development of vaccine, but to potentially curb the risk of SARS CoV-2 triggered hyperinflammatory syndrome.

\section{Case Report}

Background: Coronavirus disease (COVID-19) is a heterogeneous syndrome following severe acute respiratory syndrome coronavirus 2 (SARS-CoV-2) infection of the upper respiratory tract. In adults, the clinical condition can range from asymptomatic cases to severe acute respiratory syndrome and multiorgan dysfunction. Those at risk of developing COVID-19 related hyperinflammatory syndrome likely had an ineffective, innate immune response to this novel pathogen. Mast cells are associated with the epithelium, contributing to tissue homeostasis and epithelial barrier defense. Equipped with an array of pathogen receptors, mast cells exhibit distinct cytokine profiles, dependent on the tissue and the triggered pathogen receptors. Following viral infections, mast cells produce pro-inflammatory chemical mediators, such as interleukin-1 (IL-1) and IL-6, and these cytokines has been shown to be elevated in severe COVID19 cases. Here, we present a case of a patient with a longstanding history of signs and symptoms, worrisome for a mast cell activation syndrome (MCAS), but never had laboratory confirmation of this non-clonal mast cell activation disorder, until she contracted COVID-19. This case illustrates the need to recognize the role of mast cell activation in SARS-CoV-2 infection, not only to optimize anti-SARS-CoV-2 therapy, including the development of vaccine, but to potentially curb the risk of SARS-CoV-2 triggered hyperinflammatory syndrome.

Coronavirus disease 2019 (COVID-19) is a heterogeneous disorder following the inhalation of SARS-CoV2 particles in the nasopharyngeal cavity. Signs and symptoms range from asymptomatic or localized upper respiratory symptomatology to multi-organ dysfunction, including the lungs, kidneys, and the central nervous system. One factor implicated in the containment of SARS-CoV-2 infection is a strong innate immune response. The innate immune system of respiratory system consists of the airway epithelium 
cells are the first line of defense, which provide a mechanical barrier, the mucociliary escalator, and resident immune cells. The latter includes mast cells (MCs). Best known for their role in allergic or type 1 hypersensitivity reactions, MCs reside near the respiratory epithelial lining, situated near blood vessels, smooth muscle cells and nerves. Equipped with a wide range of surface receptors, MCs have the intrinsic capacity to identify pathogens through direct and indirect sensors, such as Toll-Like Receptors (TLR), Immunoglobulin receptors (IgR) and complement receptors (CR). Upon receptor engagement, MCs can then elucidate an array of bioactive chemicals, including interleukins, IL-1 and IL-6, heparin, histamine and proteases. Following containment of the pathogen-induced damage, MC activation must be downregulated in a timely fashion, to prevent the pathology of unnecessary or excessive inflammation. Recent studies highlight the role of endothelial and epithelial-derived products as key immunomodulators of MC activation (MCA). ${ }^{12}$

The human host response to SARS-CoV2 is heterogeneous, based on the age of the host and the initial immune response. Except for sporadic incidents of Kawasaki's disease like phenomena, most children are asymptomatic or have mild upper respiratory symptoms. In contrast, nearly one-fifth of adults will develop multi-organ dysfunction, including acute respiratory distress syndrome (ARDS), following SARSCoV2 infection. MCs have the capacity to directly detect SARS-CoV2 through pathogen associated molecular pattern (PAMPs) receptors, such as intracellular TLR 3, 4, and 7, or indirectly through IgRs or CRs. ${ }^{3}$ Dependent on state of tissue and the series of receptors triggered, SARS-CoV-2 exposure can induce distinct profiles of MC chemical mediators. Direct MC activation, via TLR engagement, can lead to the pro-inflammatory cytokines, such as IL-1 and 6. 4- 6 While indirect detection of SARS-CoV-2, through IgRs, may lead to rapid release of histamine, heparin, platelet-activating factor (PAF), proteases, as well as IL-6. Moreover, the phenotype of MCA response to SARS-CoV-2 exposure likely depends on the state of epithelial - MC interaction at the time of viral encounter. Here, we present evidence of MC activation, in a patient with known MCA disorders, who develops and promptly recovers COVID-19, following exposure to SARS-CoV-2 and associated with pathology. ${ }^{7}$ These observations suggest a prominent role of mast cells in the host response to SARS-CoV-2, and provide incentive to screen and evaluate the spectrum of MC phenotypes, which, in tum, can help tailor therapies that promote timely induction and then suppression of inflammation to this novel pathogen.

A 32-year-old Caucasian woman, with a postural orthostatic tachycardia syndrome (POTS), hypermobile type Ehlers Danlos Syndrome (hEDS), and a cluster of mast cell activation disorders, developed COVID-19 in late March 2020. With a lifelong history of recurrent infections, the patient was started on subcutaneous gammaglobulin supplement, then intravenous gammaglobulin preparations, four years prior. She has also been prescribed medications targeting the MC compartment, to due to hypersensitivity reactions impacting her skin as well as respiratory, gastro-intestinal and urogenital tracts. Her diagnoses include interstitial cystitis, hypersensitivity gastroenteritis, rhinitis, chronic spontaneous urticaria, multiple drug sensitivities, anaphylaxis and asthma. Like many patients, who present with typical features of mast cell activation (MCA) and experience some relief with medications, that targeted the MC compartment, the diagnosis of mast cell activation syndrome (MCAS) had not been confirmed, due to lack of objective 
markers of MCA. Without laboratory confirmation of persistent or recurrent MCA, many patients remain under-diagnosed and under-treated for years, receiving sub-optimal medical treatment for this hypersensitivity syndrome. In the distant past, she was also treated with low molecular weight heparin and Eliquis (apixaban) for a deep vein thrombosis (DVT) following a PICC line's traumatic placement.

With a recent move, the patient was referred to our Allergy/Immunology practice, to establish local care of her primary immunodeficiency and susceptibility to hypersensitivity reactions. Following an initial consultation, the patient was given a lab requisition to assess her immunoglobulin levels and check for validated markers of MCA, including serum tryptase and histamine. Shortly thereafter, the patient developed a low-grade fever, headache, trouble breathing, chills, body aches. She obtained a COVID nasal swab test, but the test center failed to process the sample adequately. Over the next three days, she continued the gamma-globulin infusion as well as use of histamine blockers, montelukast, ketotifen, cromolyn, and pain relievers. The fever persisted, and she also reported light sensitivity, anorexia, increased cough, nausea, vomiting, and bowel incontinence. With increasing gastrointestinal and respiratory distress, the patient sought care at he local emergency department. There, the rapid test for SARS- CoV2 was positive, and the patient was admitted for COVID-19. The hospital also processed the laboratory requisition to assess for MCAS validated markers, which revealed a serum tryptase of $8.8 \mathrm{ng} / \mathrm{ml}$. This level was above the previously reported baseline level of $5.3 \mathrm{ng} / \mathrm{ml}$, from July 2016.

Notable laboratory values on admission for COVID-19 were an elevated D-Dimer of $0.66 \mathrm{mg} / \mathrm{L}$ (greater than $0.49 \mathrm{mg} / \mathrm{L}$ ) and a decreased Prothrombin Time of 9.3 seconds (less than 12 seconds). The patient received intravenous hydration, dexamethasone, intravenous antibiotics, oxygen supplementation, and low molecular heparin. The latter addressed a newly formed DVT. The patient was able to return home after a three-day hospital stay, resuming her treatments for chronic rhinosinusitis, POTS, and MCA disorder, newly confirmed MCAS. The patient remained in quarantine for 21 days post hospitalization, along with the three other family members, who also tested positive for SARS-CoV2 infection. Her young children were asymptomatic, and her husband required supportive care for upper airway inflammation. To date, there have been no signs of long-term hauler symptomology by the patient or her family.

Table 1

\begin{tabular}{|ll|}
\hline Date & Tryptase level \\
\hline $7 / 11 / 2016$ & $5.3 \mathrm{ng} / \mathrm{ml}$ \\
\hline $3 / 12 / 2020$ & $8.8 \mathrm{ng} / \mathrm{ml}$ ** \\
\hline $5 / 6 / 2020$ & $5.6 \mathrm{ng} / \mathrm{ml}$ \\
\hline $10 / 28 / 2020$ & $6.4 \mathrm{ng} / \mathrm{ml}$ \\
\hline$* *$ during non-ICU hospitalization for COVID-19 & \\
\hline
\end{tabular}


In summary, this patient with a cluster of syndromes, including autonomic dysfunction, hypermobile Ehlers Danlos Syndrome (hEDS), and primary immunodeficiency (PID), exhibited evidence of sustained MC mediator release for the first time, following SARS-CoV2 exposure. She developed COVID-19, requiring general medical floor support for dehydration, treatment of a DVT and an asthma exacerbation. With this observation, the patient fulfilled the three criteria for the MCAS diagnosis, proposed by the World Health Organization Mast Cell Disorders working conference in 2010: (1) typical signs and symptoms of hypersensitivity reactions; (2) substantial transient increase in serum total tryptase level or an increase in other mast cell-derived mediators such as histamine or prostaglandin D2 (or their urinary metabolites); and, (3) a response of clinical symptoms to agents that attenuate mast cell mediators. ${ }^{8}$ Although still within the commercial laboratory reference range, below $11 \mathrm{ng} / \mathrm{ml}$, the patient had a marked increase in serum tryptase of $2.2 \mathrm{ng} / \mathrm{ml}$, a $+20 \%$ increase from her baseline, measured before and after SARS-CoV2 infection. er signs and symptoms, involving the skin, gastrointestinal, respiratory, and urogenital tracts, now meet the proposed criteria for MCAS, years after onset of this hypersensitivity syndrome. ${ }^{18}$

Increasing lines of evidence indicate that MCs are key coordinators of both homeostasis and inflammation, throughout the body. ${ }^{9}$ Equipped with numerous pathogen receptors and an array of potent mediators, MCs can respond to a range of infectious and non-infectious insults, with distinct profiles of chemical mediators. Direct engagement of MCs, via TLRs, results in de novo production and secretion of pro-inflammatory cytokines, such as and IL- 1 and IL-6. While indirect activation of MC, by Ig R or CR engagement, not only leads de novo production and secretion of pro-inflammatory cytokines, but rapid release of prestored proteases, heparin, platelet-activating factor, and histamine. 2 I 0 Moreover, MCs have been shown to initiate then curb different inflammatory cascades, with influence over nearby lymph node composition, the recruitment of innate and adaptive immune responses to site of infection, such as macrophages and lymphocytes, respectively, and then suppression of recruited components of the immune system, upon detection of contained or subsiding infection.

Given the key role that $\mathrm{MC}$ play in the orchestration and resolution of inflammation, $\mathrm{MC}$ activation must be tightly regulated. This is to not only coordinate a tailored, effective response to impending dangers, but also to prevent pathology associated with inappropriate or excessive inflammation, such as NC-MCAS and COVID-19 associated hyper-inflammatory syndrome. ${ }^{110}$

Recent reports highlight the role of chemical mediators derived from the surrounding epithelium and endothelium as crucial regulators of MC activation, such as IL-33 and Thymie Stromal Lymphopoietin. ${ }^{11}$

SARS-CoV-2 to angiotensin-converting enzyme 2 (ACE2) receptor on the respiratory epithelium, causing cell death. ${ }^{3}$ The subsequent epithelial cell death and tissue damage, due to virus replication, lead to the release of damage-associated molecular patterns (DAMPs), also known as alarmins. Detection of alarmins likely activates resident immune cells and triggers different inflammatory cascades, dependent of the state of the tissue and the nature of the detected insult. Recent reports highlight the role of 
macrophages and innate lymphoid cells as part of the first response to SARS-CoV-2. ${ }^{3}$ However, the strategic position of mast cells, situated below the lining of the respiratory epithelium and clustered near blood vessels and peripheral nerves, implicate the often overlooked MCs. In addition to potential recognition of SARS-CoV-2 via ACE2 receptor, MCs can directly recognize SARS-CoV2 via PAMP receptors, such as Toll-Like receptors (TLR) 3, 4 and 7, and indirectly, through IgRs and CRs. ${ }^{12}$ Depending on the recognition of SARS-COV-2 by receptors on MCs, different MC activation mediator release patterns will follow. Direct recognition of SARS CoV-2, with TLR engagement, does not trigger MC degranulation. Instead, TLR binding of SARS-CoV-2 reportedly leads to de novo production and secretion of cytokines, such as IL-1 and IL-6, as well as lipid-mediator production. Alternatively, indirect MC activation via SARSCoV-2, bound to Ig, for example, triggers MC degranulation of prestored chemical mediators, including tryptase, histamine and production of newly generated factors, such as lipid mediators and cytokines, including IL-1 and IL-6, and chemokines. ${ }^{11} 1314$

Here, we present a case of a woman with pre-existing non-clonal, MCA disorders, who exhibited a detected MC activation event following SARS-CoV-2 infection. Though she suffered with multi-organ system involvement, NC-MCA disorders and had modest relief of symptoms, with a classic allergy or type I hypersensitivity medications, this patient was not formally diagnosed with MCAS, due to lack of validated MC activation markers. Of note, lack of the MCAS diagnosis did not prevent utilization of medications that target certain MC activation pathways, specifically treatments that antagonize MC degranulation or MC mediators, such as cromolyn as well as histamine and lipid metabolites antagonists, respectively. Only upon SARS-CoV-2 infection, a sustained elevation of serum tryptase was detected, utilizing the formula of $2 \mathrm{ng} / \mathrm{ml} /+20 \%$ of the baseline serum tryptase. This increase of serum tryptase was seen, despite recent use of prednisone and her daily cocktail of histamine and leukotriene blockade. These observations suggest that MC activation participated in local tissue response to SARS-CoV-2 and was associated with COVID-19 associated hyper-inflammatory syndrome. The patient primarily required supportive care, including intravenous hydration, dexamethasone, short-acting beta-agonists, histamine blockade, leukotriene antagonists, temporary supplemental oxygen therapy via nasal cannula, and anticoagulation. Within two weeks of her hospitalization, for COVID-19 related respiratory, gastrointestinal, and hematological complications, her NC-MCAS associated symptoms returned to baseline and was subsequently started on dupilumab, to attain better asthma control. The patient's serum tryptase first tested two months later, returned to her baseline as well, thus confirming the diagnosis of non-clonal MCAS. Moreover, this patient's course, following SARS-CoV-2 infection, lends support to previous reports surmising the protective effect of pre-existing inflammatory disorders or the medications used to manage these hypersensitivity syndromes to COVID-19 associated morbidity and mortality. ${ }^{14}$

In conclusion, this case implicates a role of MC activation in SARS-CoV-2 infection and the potential to modify the development and severity of COVID-19. Among adults, COVID-19 is a heterogeneous disorder, clinically silent or mild symptoms to hyperinflammatory syndromes. This range of COVID-19 phenotypes may reflect the initial and subsequent distinct MCA profiles or phenotypes, which are influenced by signals from the local micro-environment. Depending on the state of inflammation in the affected tissue 
and the range of the receptors engaged at the time of the encounter, MCs will release distinct combination of chemical mediators, including secretion of newly produced factors, such as IL-1 and IL-6, with or without rapid release of prestored chemical mediators, such as proteases, heparin, histamine, and

bradykinin. Upon containment of the danger, MCs can release factors that attenuate tissue inflammation, such as IL-10. ${ }^{11}$ Better characterization of MC response to SARS-CoV-2 exposure may not only identify patients at risk of severe COVID-19 phenotypes, but also help tailor adjuvant therapeutic regimens to accelerate resolution of the inflammation, following clearance of the virus. This includes therapies focus on the mast cell compartment, such as vaccines adjuvants and medications, which selecting modify MC mediator release, or targeting the epithelium and cells in the surrounding microenvironment, that can suppress MC actions. Therefore, studies are needed to elaborate mast cell phenotypes, following SARS CoV-2 infection, and then optimize treatments, including medications and vaccines, which tailor the immune response to SARS CoV-2 infection and ostensibly reduce COVID-19 associated morbidity and mortality.

\section{Declarations}

Ethics approval - consent to participate was given by the patient

Consent for publication - given by all authors and the patient

Availability of data and material - The datasets used and analyzed during the current study are available from the corresponding author on reasonable request.

Competing interests - Isabelle Brock has no conflicts to declare. Anne Maitland receives compensation as part of the speakers' bureau of Sanofi Pharmaceuticals, Regeneron and Genentech. There is no conflict of interest with the observations reported in this manuscript and Dr. Maitland's affiliation.

Funding - This work received no funding.

Authors' contributions - both authors have contributed in equal parts to all parts of the study and manuscript

Acknowledgements - none

\section{References}

1. Hamilton MJ. Non-Clonal Mast Cell Activation Syndrome: A Growing body of evidence. immunol Allergy clin North Am. 2018;38(3):469-481.

2. Marshall JS. Mast-cell responses to pathogens. Nat Rev Immunol. 2004;4(10):787-799. doi:10.1038/nri1460 
3. Jafarzadeh A, Chauhan P, Saha B, Jafarzadeh S, Nemati M. Contribution of monocytes and macrophages to the local tissue inflammation and cytokine storm in COVID-19: Lessons from SARS and MERS, and potential therapeutic interventions. Life Sei.2020;257. doi:10.1016/j.Ifs.2020.118102

4. Guan W, Ni Z, Hu Y, et al. Clinical characteristics of coronavirus disease 2019 in China. N Engl J Med. 2020;382(18): 1708-1720. doi:10.1056/NEJMoa2002032

5. Qin C, Zhou L, Hu Z, et al. Dysregulation of immune response in patients with COVID- 19 in Wuhan, China. Clin Infect Dis. 2020;2019(Xx Xxxx):4-10. doi:10.1093/cid/ciaa248. Zhang W, Du RH, Li B, et al. Molecular and serological investigation of 2019-nCoV infected patients: implication of multiple shedding routes. Emerg Microbes Infect. 2020;9(1):386-389. doi:10.1080/22221751.2020.1729071

6. Coperchini F, Chiovato L, Croce L, Magri F, Rotondi M. The cytokine storm in COVID-19: An overview of the involvement of the chemokine/chemokine-receptors. Cytokine208 Growth Factor Rev. 2020; (January).

7. Theoharides TC, Valent P, Akin C. Mast Cells, Mastocytosis, and Related Disorders. N Engl J Med. 2 015;373(2):163-172 . doi:10.1056/nejmra1409760

8. Campillo-Navarro M, Chavez-Blanco AD, Wong-Baeza I, et al. Mast cells in lung homeostasis: Beyond type i hypersensitivity. Corp Commun. 2005;10(2):115-123.

doi:10.2174/1573398x10666141024220151

9. Jimenez-Rodriguez TW, Garcia-Neuer M, Alenazy LA, Castells M. Anaphylaxis in the $21^{\text {st }}$ century: Phenotypes, endotypes, and biomarkers. J Asthma Allergy. 2018;11:121-142. doi:10.2147/JAA.SI59411

10. Kritas SK, Ronconi G, Caraffa A, Gallenga CE, Ross R, Conti P. Mast cells contribute to coronavirusinduced inflammation: New anti-inflammatory strategy. J Bio! Regul Homeost Agents. 2020;34(1):914. doi:10.23812/20-Editorial-Kritas

11. Sallenave JM, Guillot L. Innate Immune Signaling and Proteolytic Pathways in the Resolution or Exacerbation of SARS-CoV-2 in Covid-19: Key Therapeutic Targets? Front Immunol. 2020;11(May):19. doi:10.3389/fimmu.2020.01229

12. Theoharides TC. Potential Association of Mast Cells with COVID-19. Ann Allergy, Asthma Immunol. Published online 2020. doi:10 .1016/j.anai.2020.11.003

13. Theoharides TC. COVID-19, pulmonary mast cells, cytokine storms, and beneficial actions of luteolin. BioFactors. 2020;46(3):306-308.doi:10.1002/biof.1633 